\title{
Multirotor Aerial Vehicle modeling in Modelica
}

\author{
Muhamed Kuric $^{1}$ Nedim Osmic $^{1} \quad$ Adnan Tahirovic $^{1}$ \\ ${ }^{1}$ Department of Automatic Control and Electronics \\ Faculty of Electrical Engineering \\ University of Sarajevo \\ Zmaja od Bosne bb, 71000 Sarajevo, Bosnia and Herzegovina \\ \{muhamed.kuric, nedim.osmic, adnan.tahirovic\}@etf.unsa.ba
}

\begin{abstract}
This paper presents a generalized Multirotor Aerial Vehicle (MAV) modeling framework which includes rigid body dynamics, gyroscopic effect and motor dynamics. We illustrate how this model can be used to derive any MAV platform constructed with an arbitrary number of rotors by using the quadrotor case as an example. Based on this result, we design the first Modelica-based MAV simulator. We validate the proposed design by using a simple altitude and attitude stabilization control system through a Modelica simulation setup.
\end{abstract}

Keywords: Multirotor Aerial Vehicle, Modeling, Modelica

\section{Introduction}

Technological advancements in recent years, including the miniaturization in battery, sensor and actuation technologies, as well as the availability of low cost high performance computing boards have enabled the genesis of intelligent autonomous flying machines. The most popular class of this machines are the so-called Multirotor Aerial Vehicles (MAVs) which represent motorized rotorcrafts that have favourable dynamical properties and can achieve small geometries. MAVs and especially the quadrotor configuration are now the de facto standard research platforms for aerial robotics with many potential applications including search and rescue in indoor and outdoor environments (Tomic et al., 2012), precision agriculture (Zhang and Kovacs, 2012), aerial construction (Lindsey et al., 2011; Willmann et al., 2012), inspection and maintenance (Mellinger et al., 2011; Jimenez-Cano et al., 2013), environmental monitoring (Alexis et al., 2009), exploration and mapping (Fraundorfer et al., 2012), aerial transportation (Michael et al., 2011; Mellinger et al., 2013) and swarming (Kushleyev et al., 2013).

Due to this growing interest, there have emerged multiple MAV simulation platforms mainly in MATLAB and ROS with notable examples being (Bresciani, 2008) and (Furrer et al., 2016), respectively. Both provide simulation for MAV dynamics (with the former covering only the quadrotor case) and sensors, and the latter having a less user-friendly interface via pure code and configuration. To the best of our knowledge, there are no existing MAV simulation platforms within the Modelica commu- nity.

Our paper gives a simple way of deriving a proper dynamical model for a MAV constructed with an arbitrary number of rotors by using a generalized MAV model. Based on this paradigm, we also present a Modelica simulator that can be used for multirotor aerial vehicles. To the best of the authors' knowledge, this is the first Modelicabased MAV simulator available within the Modelica community.

The remainder of the paper is organized as follows. Section 2 describes how generalized MAV dynamics can be derived and how an appropriate dynamical model can be extracted for a quadrotor based MAV. In Section 3, we describe necessary classes to design the Modelica-based simulator for MAVs, while in Section 4, we validate the results throughout a simple altitude and attitude stabilization control system. Concluding remarks are presented in Section 5 .

\section{MAV dynamics}

A large number of papers address MAV modeling putting the focus mostly on the quadrotor case. Noteworthy classical contributions include (Altug et al., 2002), (Hamel et al., 2002), (Pounds et al., 2002) and (Bouabdallah et al., 2004a). More recent examples of very detailed quadrotor and octorotor modeling are presented in (Bangura and Mahony, 2012) and (Osmic et al., 2016), respectively. To the best of our knowledge, one of the most complete work regarding MAVs can be found in (Mahony et al., 2012), where the authors have derived MAV dynamics, included advanced state estimation, control and motion planning algorithms and therefore provided full system autonomy.

In this section, we will describe the dynamical model of the quadrotor, which is frequently considered to be the standard research platform for MAVs due to its simple construction and purposeful functionality. We use the results and nomenclature from (Osmic et al., 2016) and show that only minor changes are necessary to apply the final octocopter model presented in (Osmic et al., 2016) to any $\mathrm{MAV}$, including also the quadrotor case.

\subsection{MAV rigid body dynamics}

In order to model the dynamics of any mobile robot it is common to define two frames of reference. A body fixed 
frame $\{o\}$ is attached to the robots center of mass and all sensory data is measured with respect to this frame, while a ground fixed frame $\{g\}$ is used to define workspace goals in a intuitive and user-friendly manner. The body fixed and ground fixed frame represent right-handed Cartesian coordinate systems and are usually referred to as the local and global coordinate system, respectively.

Workspace goals can be defined in terms of global position coordinates $x, y$ and $z$ and orientation coordinates $\phi$, $\theta$ and $\psi$ (see Fig. 1), where positive directions of $\phi, \theta$ and $\psi$ are chosen according to the right-hand rule. Therefore, the position vector $\boldsymbol{x}=\left[\begin{array}{ll}x & y\end{array}\right]^{T}$ and the orientation vector $\Psi=\left[\begin{array}{lll}\phi & \theta & \psi\end{array}\right]^{T}$ can completely determine the vehicle's location in the workspace. As shown in Fig. 2, the local coordinates are described by the linear velocities $u, v$ and $w$ and the angular velocities $P, Q, R$. The positive directions of the angular velocities $P, Q$ and $R$ are also chosen according to the right-hand rule and therefore coincide with the positive directions of $\phi, \theta$ and $\psi$. Both linear and angular velocity coordinates can also be expressed in compact vector form as $\boldsymbol{v}=\left[\begin{array}{lll}u & v & w\end{array}\right]^{T}$ and $\boldsymbol{P}=\left[\begin{array}{lll}P & Q & R\end{array}\right]^{T}$, respectively.

Forces and torques which act on MAV are shown in Fig. 3. The thrust $T$ is a force that acts towards the positive direction of the $Z$ axis of the local coordinate system $\{o\}$, while the force $G$ represents the gravitational force acting towards the negative direction of the $Z_{B}$ axis of the global coordinate system $\{g\} . \tau_{x}, \tau_{y}$ and $\tau_{z}$ represent the torques that move the vehicle around the $X, Y$ and $Z$ axes of the local coordinate system, respectively, and can be compactly denoted as $\boldsymbol{\tau}=\left[\begin{array}{lll}\tau_{x} & \tau_{y} & \tau_{z}\end{array}\right]^{T}$. Their positive direction is also chosen to coincide with the positive directions of the angular velocities $P, Q$ and $R$.

We can now describe the rigid body dynamics of any

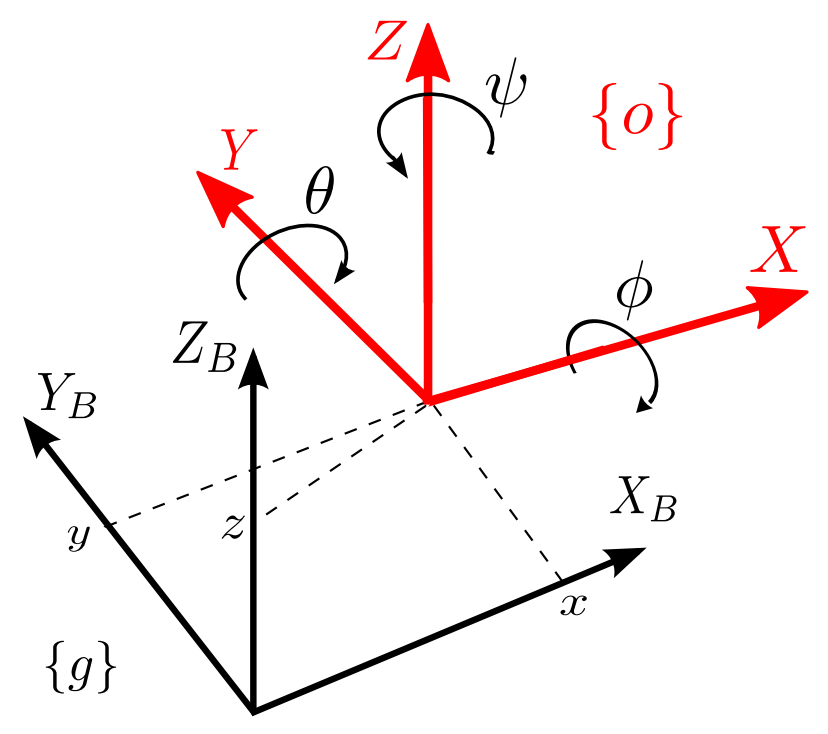

Figure 1. Global coordinates
MAV in accordance to the results presented in (Osmic et al., 2016). The kinematic model of the linear motion is given as

$$
\dot{\boldsymbol{x}}=\boldsymbol{R}(\phi, \theta, \psi) \boldsymbol{v},
$$

where $\boldsymbol{R}(\phi, \theta, \psi)$ is the total rotation matrix which for the ZYX Euler convention has the form

$$
\begin{aligned}
& \boldsymbol{R}(\phi, \theta, \psi)=R(Z, \psi) R(Y, \theta) R(X, \phi) \\
& {\left[\begin{array}{ccc}
c_{\psi} c_{\theta} & c_{\psi} s_{\theta} s_{\phi}-s_{\psi} c_{\phi} & c_{\psi} s_{\theta} c_{\phi}+s_{\psi} s_{\phi} \\
s_{\psi} c_{\theta} & s_{\psi} s_{\theta} s_{\phi}+c_{\psi} c_{\phi} & s_{\psi} s_{\theta} c_{\phi}-c_{\psi} s_{\phi} \\
-s_{\theta} & c_{\theta} s_{\phi} & c_{\theta} c_{\phi}
\end{array}\right]}
\end{aligned}
$$

and the elementary rotation matrices $R(Z, \psi), R(Y, \theta)$ and $R(X, \phi)$ are defined as

$$
\begin{aligned}
& R(X, \phi)=\left[\begin{array}{ccc}
1 & 0 & 0 \\
0 & c_{\phi} & -s_{\phi} \\
0 & s_{\phi} & c_{\phi}
\end{array}\right], \\
& R(Y, \theta)=\left[\begin{array}{ccc}
c_{\theta} & 0 & s_{\theta} \\
0 & 1 & 0 \\
-s_{\theta} & 0 & c_{\theta}
\end{array}\right], \\
& R(Z, \psi)=\left[\begin{array}{ccc}
c_{\psi} & -s_{\psi} & 0 \\
s_{\psi} & c_{\psi} & 0 \\
0 & 0 & 1
\end{array}\right] .
\end{aligned}
$$

The kinematic model of the angulator motion can be described by

$$
\dot{\boldsymbol{\Psi}}=\boldsymbol{R}_{A}^{-1}(\phi, \theta, \psi) \boldsymbol{P},
$$

where the matrix $\boldsymbol{R}_{A}^{-1}(\phi, \theta, \psi)$ for the ZYX Euler convention is

$$
\boldsymbol{R}_{A}^{-1}(\phi, \theta, \psi)=\left[\begin{array}{ccc}
1 & s_{\phi} t_{\theta} & c_{\phi} t_{\theta} \\
0 & c_{\phi} & -s_{\phi} \\
0 & \frac{s_{\phi}}{c_{\theta}} & \frac{c_{\phi}}{c_{\theta}}
\end{array}\right] .
$$

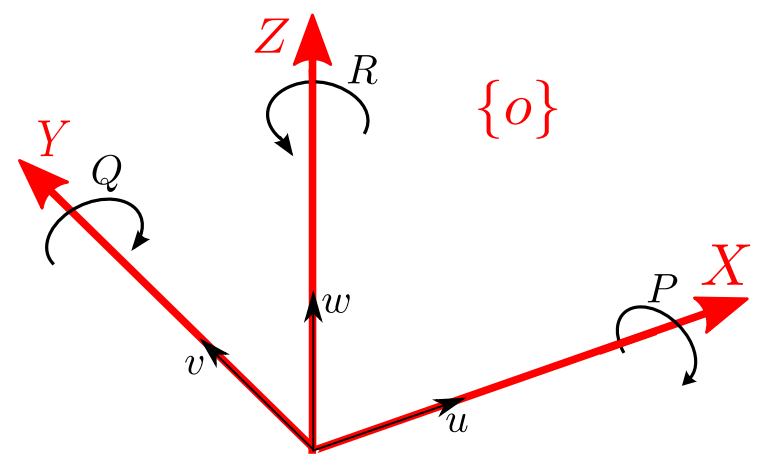

Figure 2. Local coordinates 
The dynamic model of the linear motion can be represented by the following equation

$$
\dot{\boldsymbol{v}}=\left[\begin{array}{c}
0 \\
0 \\
\frac{T}{m_{o}}
\end{array}\right]+g\left[\begin{array}{c}
s_{\theta} \\
-s_{\phi} c_{\theta} \\
-c_{\phi} c_{\theta}
\end{array}\right]-\boldsymbol{S} \boldsymbol{v}
$$

where $m_{o}$ is the total mass of the MAV and the matrix $S$ is formed as

$$
\boldsymbol{S}=\left[\begin{array}{ccc}
0 & -R & Q \\
R & 0 & -P \\
-Q & P & 0
\end{array}\right] .
$$

Finally, the dynamic model of the angular motion can be catched with

$$
\dot{\boldsymbol{P}}=\boldsymbol{J}^{-1}(\boldsymbol{\tau}-\boldsymbol{S J P}),
$$

where $\boldsymbol{J}$ is a $3 \times 3$ matrix representing the inertia tensor of the MAV.

\subsection{Quadrotor modeling}

To tailor the previously derived MAV model to the quadrotor case we need to derive the inertia tensor $\boldsymbol{J}$, and define the thrust $T$ and the torque vector $\tau$. Since all of these quantities depend on the MAV's geometry, we consider a quadrotor case shown in Fig. 4 along with its simplified geometry illustrated in Fig. 5, where the length of the four arms is $l$, a hardware support plate is modeled as solid sphere of mass $M$ having a radius $r$, and the four motors constructed with fixed pitch propellers are modelled as particles with mass $m$.

The axes of the local coordinate system, as shown in Fig. 4, represent principal axes of inertia, where the inertia

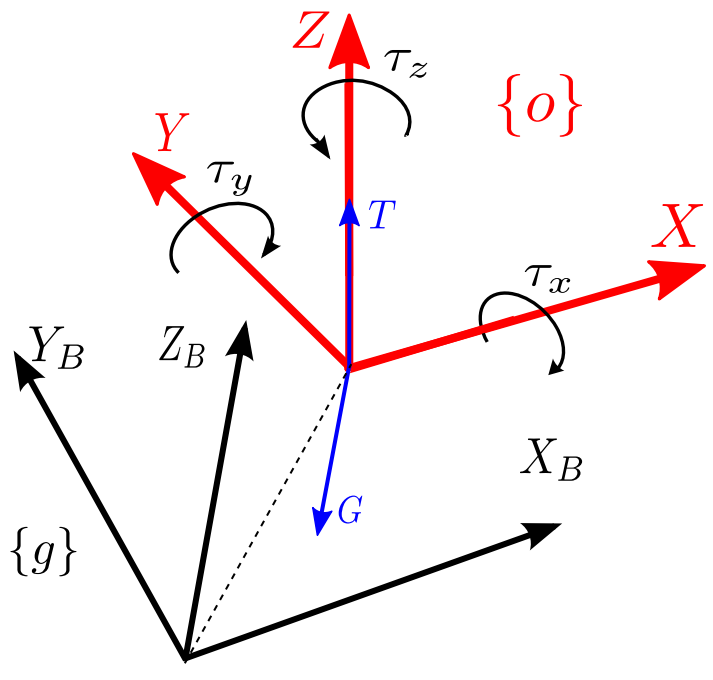

Figure 3. Forces and torques acting on the system tensor matrix has the diagonal form

$$
\boldsymbol{J}=\left[\begin{array}{ccc}
I_{x x} & 0 & 0 \\
0 & I_{y y} & 0 \\
0 & 0 & I_{z z}
\end{array}\right],
$$

and $I_{x x}, I_{y y}, I_{z z}$ being the moments of inertia around the $X$, $Y$ and $Z$ axes of the local coordinate system, respectively. These components can be derived via the Huygens-Steiner theorem (Morin, 2008) as

$$
I_{x x}=I_{y y}=\frac{2 M r^{2}}{5}+2 m l^{2}
$$

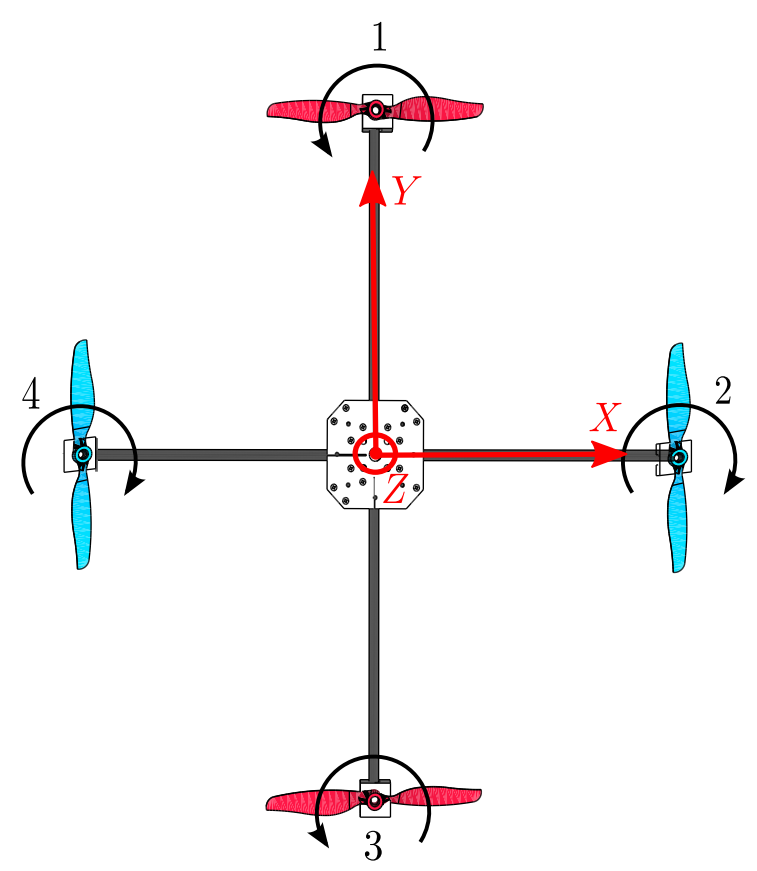

Figure 4. Quadrotor geometry

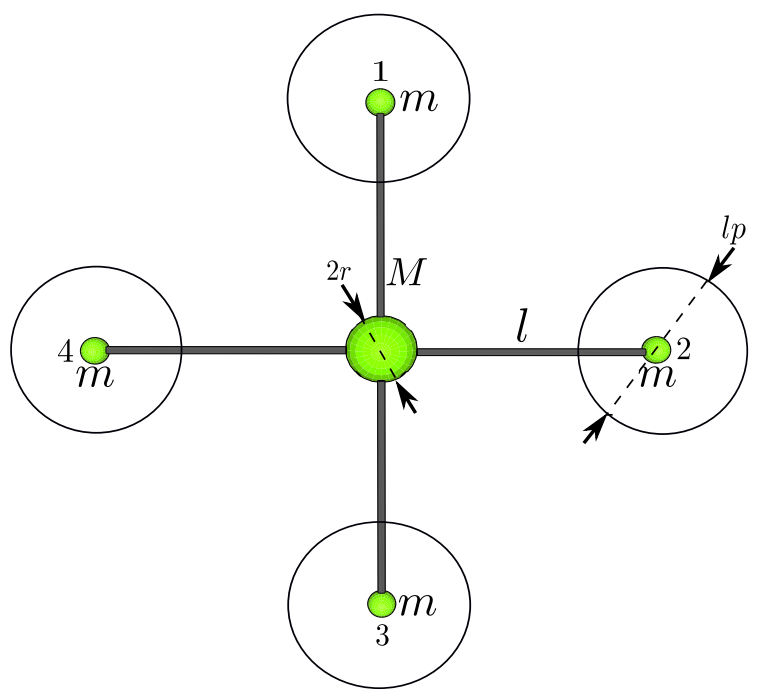

Figure 5. Quadrotor simplified geometry 
and

$$
I_{z z}=\frac{2 M r^{2}}{5}+4 m l^{2}
$$

In order to derive the thrust $T$ and the $\tau_{x}$ and $\tau_{y}$ components of the torque vector $\tau$, we will consider the rotor forces acting on the quadrotor system as depicted in Fig. 6. Thus $T, \tau_{x}$ and $\tau_{y}$ are given as follows

$$
\begin{gathered}
T=F_{1}+F_{2}+F_{3}+F_{4}, \\
\tau_{x}=l\left(F_{1}-F_{3}\right), \\
\tau_{y}=l\left(F_{4}-F_{2}\right) .
\end{gathered}
$$

In accordance to the work presented in (Mahony et al., 2012), the rotor forces $F_{i}(i=\overline{1 . .4})$ can be approximated as

$$
F_{i}=b \Omega_{i}^{2}(i=\overline{1 . .4}),
$$

where $b\left[\frac{\mathrm{Ns}^{2}}{\mathrm{rad}^{2}}\right]$ is the rotor thrust constant and $\Omega_{i}\left[\frac{\mathrm{rad}}{\mathrm{s}}\right]$ is the angular velocity of the $i$-th rotor. Combining eqs. (14), (15), (16) and (17) yields

$$
\begin{gathered}
T=b\left(\Omega_{1}^{2}+\Omega_{2}^{2}+\Omega_{3}^{2}+\Omega_{4}^{2}\right), \\
\tau_{x}=b l\left(\Omega_{1}^{2}-\Omega_{3}^{2}\right)
\end{gathered}
$$

and

$$
\tau_{x}=b l\left(\Omega_{4}^{2}-\Omega_{2}^{2}\right) .
$$

The torque $\tau_{z}$ is a consequence of Newton's third law and can be formed as

$$
\tau_{z}=-M_{1}+M_{2}-M_{3}+M_{4},
$$

where $M_{i}(i=\overline{1 . .4})$ is the counter induced torque of the $i$-th rotor. According to (Mahony et al., 2012) the counter torque can approximated as

$$
M_{i}=d \Omega_{i}^{2}(i=\overline{1 . .4}),
$$

where $d\left[\frac{\mathrm{Nms}^{2}}{\mathrm{rad}^{2}}\right]$ is the rotor drag constant. Combining equations (21) and (22) yields

$$
\tau_{z}=d\left(-\Omega_{1}^{2}+\Omega_{2}^{2}-\Omega_{3}^{2}+\Omega_{4}^{2}\right) .
$$

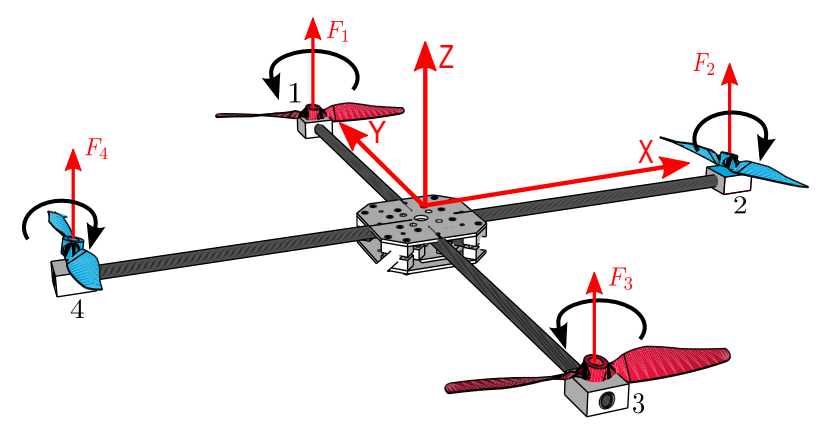

Figure 6. Rotor forces acting on the quadrotor system
Finally, we can represent the system actuation via matrix equation

$$
\left[\begin{array}{l}
T \\
\tau
\end{array}\right]=A \boldsymbol{\Omega}_{\boldsymbol{s}},
$$

where $\boldsymbol{A}$ is the actuation matrix

$$
\boldsymbol{A}=\left[\begin{array}{cccc}
b & b & b & b \\
b l & 0 & -b l & 0 \\
0 & -b l & 0 & b l \\
-d & d & -d & d
\end{array}\right],
$$

and $\boldsymbol{\Omega}_{\boldsymbol{s}}$ is the squared rotor velocity vector defined as

$$
\boldsymbol{\Omega}_{\boldsymbol{s}}=\left[\begin{array}{llll}
\Omega_{1}^{2} & \Omega_{2}^{2} & \Omega_{3}^{2} & \Omega_{4}^{2}
\end{array}\right]^{T} .
$$

It is evident from this result that any MAV can be modelled by choosing the appropriate inertia tensor $\boldsymbol{J}$ and actuation matrix $\boldsymbol{A}$ as parameters, and picking the squared rotor velocity vector $\boldsymbol{\Omega}_{\boldsymbol{s}}$ of the right size as a system input. For any MAV constructed with $n \geq 4$ rotors, the actuation matrix has the dimension $4 \times n$ and the squared rotor velocity vector $\boldsymbol{\Omega}_{\boldsymbol{s}}$ has the length $n$.

Moreover, we can include the gyroscopic effect in the dynamic model of the angular motion given by eq. (10) as

$$
\dot{\boldsymbol{P}}=\boldsymbol{J}^{-1}\left(\boldsymbol{\tau}-\boldsymbol{S J P}-\boldsymbol{S}\left[\begin{array}{c}
0 \\
0 \\
I_{z z m} W_{g}
\end{array}\right]\right),
$$

where $I_{z z m}$ is the rotor moment of inertia and $W_{g}$ is the gyroscopic term given as

$$
W_{g}=-\Omega_{1}+\Omega_{2}-\Omega_{3}+\Omega_{4}
$$

for the quadrotor case. In order to generalize the gyroscopic term for any MAV configuration, it is more appropriate to choose the rotor velocity vector $\boldsymbol{\Omega}$

$$
\boldsymbol{\Omega}=\left[\begin{array}{llll}
\Omega_{1} & \Omega_{2} & \Omega_{3} & \Omega_{4}
\end{array}\right]^{T}
$$

as system input and express the gyroscopic term as

$$
W_{g}=\operatorname{sign}\left(\boldsymbol{A}_{\boldsymbol{z}}\right) \boldsymbol{\Omega},
$$

where $\boldsymbol{A}_{\boldsymbol{z}}$ is the fourth row of the actuation matrix $\boldsymbol{A}$, while the squared rotor velocity vector $\boldsymbol{\Omega}_{s}$ can easily be computed by calculating the element-wise square of the vector $\Omega$. 


\subsection{Motor dynamics}

Each rotor of a quadrotor MAV is driven by a DC motor. Therefore, in order to obtain a precise MAV model, it is inevitable to include the motor dynamics to address effects like motor response time, saturation and power consumption. In accordance to the work presented in (Osmic et al., 2016), a simplified model can be used for this purpose which is given by

$$
I_{z z m} \dot{\Omega}_{i}+\frac{K_{m} K_{e}}{R} \Omega_{i}=\frac{K_{m}}{R} v_{i}-\tau_{l i}, \quad i=\overline{1 . .4},
$$

where $K_{m}\left[\frac{N m}{A}\right]$ is the mechanical motor constant, $K_{e}\left[\frac{V s}{\mathrm{rad}}\right]$ being the electrical motor constant, $R$ denotes the armature resistance, $v_{i}$ is the armature voltage, with $\tau_{l i}$ being the load torque of the $i$-th motor. The load torque is the aerodynamic drag which can be computed as

$$
\tau_{l i}=d \Omega_{i}^{2}, \quad i=\overline{1 . .4} .
$$

The input voltage of each motor is saturated by the following box constraint

$$
0 \leq v_{i} \leq v_{\max }, \quad i=\overline{1 . .4}
$$

where $v_{\max }$ is the maximum armature voltage, and consequently the angular velocity of each rotor is also box constrained by

$$
0 \leq \Omega_{i} \leq \Omega \max , \quad i=\overline{1 . .4}
$$

where $\Omega_{\max }$ is the maximum angular velocity which can be easily computed from the stationary state of the motor dynamic model given by (31).

Finally, the rotor moment of inertia $I_{z z m}$ can be approximately calculated as

$$
I_{z z m}=\frac{m_{p} l_{p}^{2}}{12},
$$

where $m_{p}$ is the mass and $l_{p}$ being the length of the rotor.

\section{Modelica design}

In order to provide a greater end-user utilization, we designed the following Modelica blocks / classes:

- MavBase

- MavSimple

- MavFull

The MaveBase block, as shown in Fig. 7, is the simplest and it models the rigid body dynamics including the gyroscopic effect covered with eqs. (1), (6), (8) and (27). Its inputs are the generalized forces acting on the system and the outputs are the global coordinates of the system and its derivations.

The MavSimple block, as shown in Fig. 8, extends the MavBase block with the actuation model given by eq. (24)

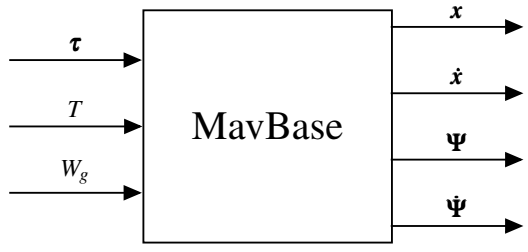

Figure 7. MavBase block

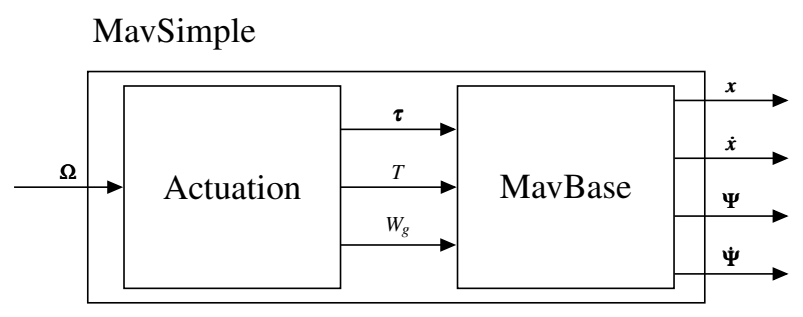

Figure 8. MavSimple block

MavFull

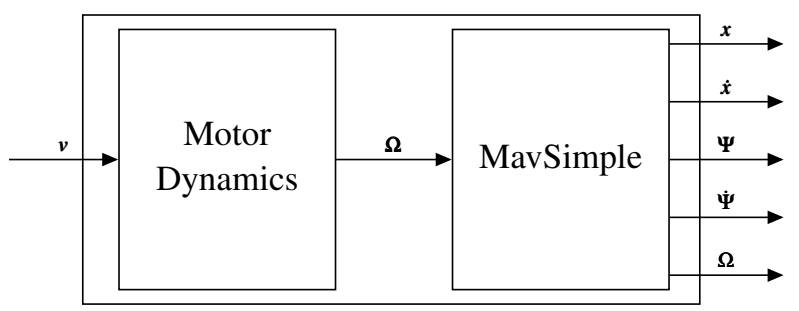

Figure 9. MavFull block

with the input being the angular velocity vector $\boldsymbol{\Omega}$ and the outputs being the global coordinates of the system and its derivations.

Finally, the MavFull block, as shown in figure 9, provides the greatest level of detail. It extends the MavSimple block and adds the motor dynamics (31) to the model. The block input is the motor voltage vector $\boldsymbol{v}$ with the outputs being the global coordinates of the system and its derivations, as well as the angular velocity vector $\boldsymbol{\Omega}$. The angular velocity vector as system output is necessary to provide motor level control possibilities.

The parameters of the blocks are given in Table 1, 2 and 3, and their default values match the AscTec Pelican quadrotor (AscTec, 2016).

Table 1. MavFull block parameters

\begin{tabular}{|c|c|c|c|}
\hline Parameter & Value & Unit & Description \\
\hline \hline$R$ & 0.1107 & $\Omega$ & Resistance \\
\hline$K_{m}$ & 0.01 & $\frac{\mathrm{Nm}}{\mathrm{A}}$ & Motor size constant \\
\hline$K_{e}$ & 0.01 & $\frac{\mathrm{Vs}}{\mathrm{rad}}$ & Motor velocity constant \\
\hline$v_{\max }$ & 11.1 & $V$ & Maximum voltage \\
\hline$\Omega_{0}$ & 569.3572 & $\frac{\mathrm{rad}}{\mathrm{sec}}$ & Initial angular velocity \\
\hline
\end{tabular}


Table 2. MavBase block parameters

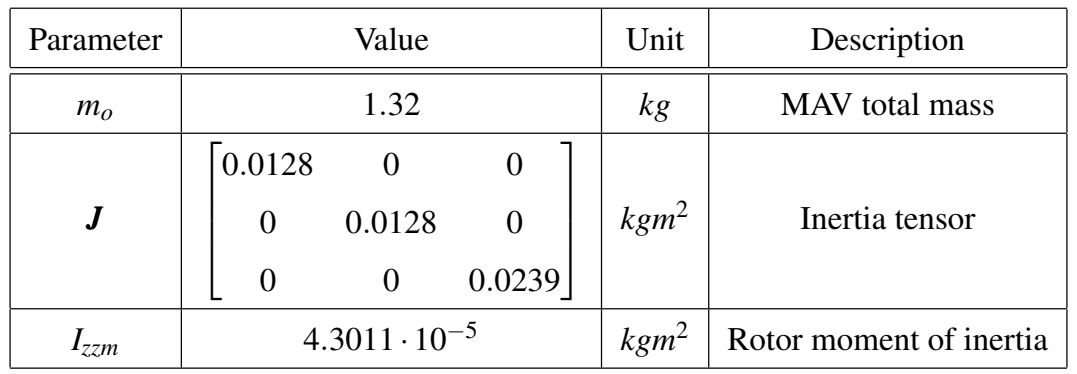

Table 3. MavSimple block parameters

\begin{tabular}{|c|c|c|c|c|c|c|}
\hline Parameter & \multicolumn{4}{|c|}{ Value } & Unit & Description \\
\hline$n$ & \multicolumn{4}{|c|}{4} & & Input size \\
\hline$b$ & \multicolumn{4}{|c|}{$9.9865 \cdot 10^{-6}$} & $\frac{N s^{2}}{\mathrm{rad}^{2}}$ & Aerodynamic thrust constant \\
\hline$d$ & \multicolumn{4}{|c|}{$1.5978 \cdot 10^{-7}$} & $\frac{\mathrm{Nms}^{2}}{\mathrm{rad}^{2}}$ & Aerodynamic drag constant \\
\hline$A$ & {$\left[\begin{array}{c}b \\
0.211 \cdot b \\
0 \\
-d\end{array}\right.$} & $\begin{array}{c}b \\
0 \\
-0.211 \cdot b \\
d\end{array}$ & $\begin{array}{c}b \\
-0.211 \cdot b \\
0 \\
-d\end{array}$ & $\begin{array}{c}b \\
0 \\
0.211 \cdot b \\
d\end{array}$ & & Actuation matrix \\
\hline
\end{tabular}
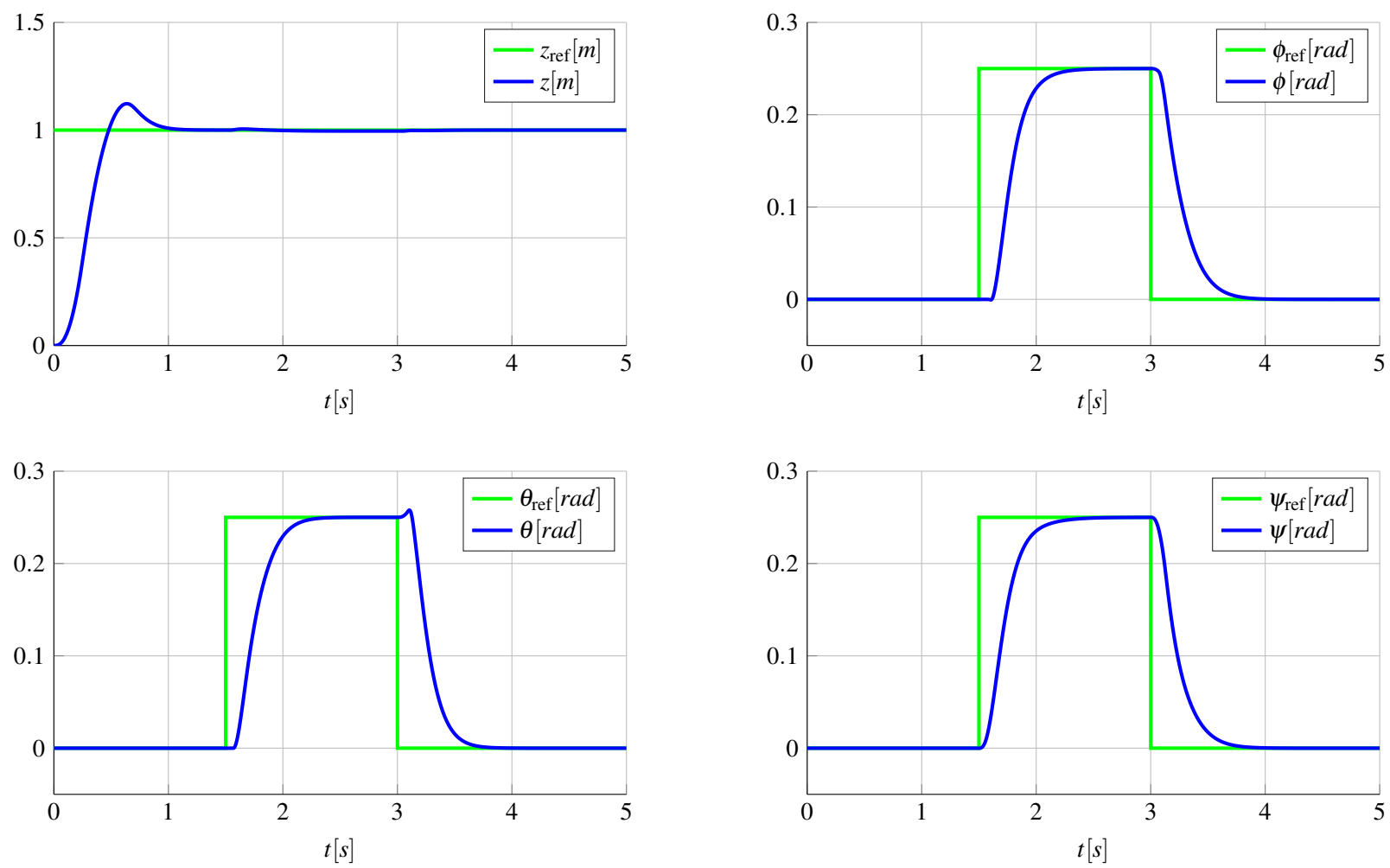

Figure 10. Altitude and attitude control simulation results 


\section{Simulation results}

A simple altitude and attitude control system was designed in order to validate the designed classes. Altitude and attitude control simulation results are presented in Fig. 10. We notice that the system states have been stabilized within 1 second and that only very minor overshoots are present in the altitude $z$ and the pitch $\theta$.

The simulation example shows that the control results are very satisfactory, in particular the system suffers only a minor loss in altitude during the challenging reference orientation maneuver, which can be considered excellent control behaviour. Additionally, the simulation results are very similar to those obtained in (Bouabdallah et al., 2004b) and (Osmic et al., 2016) which suggests that the model derivation in this paper is correct.

\section{Conclusion}

This paper described how a generalized MAV modeling framework can be used to obtain any MAV model. A quadrotor based MAV was presented as an example, and the final model was formed by using its rigid body dynamics, the gyroscopic effect that influences the vehicles motion, and appropriate motor dynamics. To model the dynamics of any given MAV platform, it was shown that is only required to choose adequate parameter values, which correspond to the vehicle of interest, and inject them into the generalized MAV model.

Based on the presented generalized MAV model derivation, we have designed the following Modelica classes: MavBase, MavSimple and MavFull. MavBase represents the rigid body dynamics of the MAV including the gyroscopic effect. MavSimple extends the MavBase class and adds system actuation, while MavFull extends MavSimple with motor dynamics. These classes can be used to simulate the dynamic behaviour of any MAV within Modelica to any required level of detail, and thus providing similar functionalities as the Gazebo simulator RotorS (Furrer et al., 2016) which is frequently used for this purposes, but with a more user friendly interface.

Finally, we have validated the designed Modelica simulator through a simple altitude and attitude stabilization control system. Namely, we have obtained very similar control results like those currently present in the state of the art, which suggests that the generalized model derived and the MAV simulator designed in this paper are correct.

\section{References}

K Alexis, G Nikolakopoulos, A Tzes, and L Dritsas. Coordination of helicopter UAVs for aerial forest-fire surveillance. In Applications of intelligent control to engineering systems, pages 169-193. Springer, 2009.

Erdinc Altug, James P Ostrowski, and Robert Mahony. Control of a quadrotor helicopter using visual feedback. In Robotics and Automation, 2002. Proceedings. ICRA'02. IEEE International Conference on, volume 1, pages 72-77. IEEE, 2002. doi:10.1109/ROBOT.2002.1013341.
AscTec. Ascending technologies, gmbh, 2016. URL http: //www.asctec.de/.

Moses Bangura and Robert Mahony. Nonlinear dynamic modeling for high performance control of a quadrotor. In Australasian conference on robotics and automation, pages 1-10, 2012.

Samir Bouabdallah, Pierpaolo Murrieri, and Roland Siegwart. Design and control of an indoor micro quadrotor. In Robotics and Automation, 2004. Proceedings. ICRA'04. 2004 IEEE International Conference on, volume 5, pages 4393-4398. IEEE, 2004a. doi:10.1109/ROBOT.2004.1302409.

Samir Bouabdallah, Andre Noth, and Roland Siegwart. PID vs LQ control techniques applied to an indoor micro quadrotor. In Intelligent Robots and Systems, 2004.(IROS 2004). Proceedings. 2004 IEEE/RSJ International Conference on, volume 3, pages 2451-2456. IEEE, 2004b.

Tammaso Bresciani. Modelling, identification and control of a quadrotor helicopter. MSc Theses, 2008.

Friedrich Fraundorfer, Lionel Heng, Dominik Honegger, Gim Hee Lee, Lorenz Meier, Petri Tanskanen, and Marc Pollefeys. Vision-based autonomous mapping and exploration using a quadrotor MAV. In Intelligent Robots and Systems (IROS), 2012 IEEE/RSJ International Conference on, pages 4557-4564. IEEE, 2012.

Fadri Furrer, Michael Burri, Markus Achtelik, and Roland Siegwart. RotorS-A Modular Gazebo MAV Simulator Framework. In Robot Operating System (ROS), pages 595-625. Springer, 2016.

Tarek Hamel, Robert Mahony, Rogelio Lozano, and James Ostrowski. Dynamic modelling and configuration stabilization for an x4-flyer. IFAC Proceedings Volumes, 35(1):217-222, 2002. doi:10.3182/20020721-6-ES-1901.00848.

AE Jimenez-Cano, Jesús Martin, Guillermo Heredia, Aníbal Ollero, and R Cano. Control of an aerial robot with multi-link arm for assembly tasks. In Robotics and Automation (ICRA), 2013 IEEE International Conference on, pages 4916-4921. IEEE, 2013.

Alex Kushleyev, Daniel Mellinger, Caitlin Powers, and Vijay Kumar. Towards a swarm of agile micro quadrotors. $A u$ tonomous Robots, 35(4):287-300, 2013.

Quentin Lindsey, Daniel Mellinger, and Vijay Kumar. Construction of cubic structures with quadrotor teams. Proc. Robotics: Science \& Systems VII, 2011.

Robert Mahony, Vijay Kumar, and Peter Corke. Multirotor aerial vehicles: Modeling, estimation, and control of quadrotor. IEEE robotics \& automation magazine, 19(3):20-32, 2012. doi:10.1109/MRA.2012.2206474.

Daniel Mellinger, Quentin Lindsey, Michael Shomin, and Vijay Kumar. Design, modeling, estimation and control for aerial grasping and manipulation. In Intelligent Robots and Systems (IROS), 2011 IEEE/RSJ International Conference on, pages 2668-2673. IEEE, 2011. 
Daniel Mellinger, Michael Shomin, Nathan Michael, and Vijay Kumar. Cooperative grasping and transport using multiple quadrotors. In Distributed autonomous robotic systems, pages 545-558. Springer, 2013.

Nathan Michael, Jonathan Fink, and Vijay Kumar. Cooperative manipulation and transportation with aerial robots. Autonomous Robots, 30(1):73-86, 2011.

David Morin. Introduction to classical mechanics: with problems and solutions. Cambridge University Press, 2008.

Nedim Osmic, Muhamed Kuric, and Ivan Petrovic. Detailed octorotor modeling and PD control. In Systems, Man, and Cybernetics (SMC), 2016 IEEE International Conference on, pages 2182-2189, 2016.

Paul Pounds, Robert Mahony, Peter Hynes, and Jonathan M Roberts. Design of a four-rotor aerial robot. In Proceedings of the 2002 Australasian Conference on Robotics and Automation (ACRA 2002), pages 145-150. Australian Robotics \& Automation Association, 2002.

Teodor Tomic, Korbinian Schmid, Philipp Lutz, Andreas Domel, Michael Kassecker, Elmar Mair, Iris Lynne Grixa, Felix Ruess, Michael Suppa, and Darius Burschka. Toward a fully autonomous UAV: Research platform for indoor and outdoor urban search and rescue. IEEE robotics \& automation magazine, 19(3):46-56, 2012.

Jan Willmann, Federico Augugliaro, Thomas Cadalbert, Raffaello D'Andrea, Fabio Gramazio, and Matthias Kohler. Aerial robotic construction towards a new field of architectural research. International journal of architectural computing, 10(3):439-459, 2012.

Chunhua Zhang and John M Kovacs. The application of small unmanned aerial systems for precision agriculture: a review. Precision agriculture, 13(6):693-712, 2012. 